\title{
Synthesis of a new diaazacrown ether compound interconnected with an azacrown ether and decorated with a long lipophilic chain
}

Jérémy Dhainaut, ${ }^{1,2}$ Alban Chappaz, ${ }^{1,3}$ Didier Bernard, ${ }^{1}$ Hélène Chaumeil, ${ }^{3 *}$ T. Jean Daou, ${ }^{2}$ Albert Defoin, ${ }^{3}$ Loïc Rouleau, ${ }^{1}$ Nicolas Bats, ${ }^{1}$ Bogdan Harbuzaru, ${ }^{1}$ Joël Patarin. ${ }^{2}$

${ }^{1}$ IFP Energies nouvelles, Rond-point de l'échangeur de Solaize, 69360 Solaize, France

${ }^{2}$ Université de Haute-Alsace (UHA), CNRS, Equipe Matériaux à Porosité Contrôlée (MPC) Institut de Science des Matériaux de Mulhouse (IS2M) UMR 7361, Institut Jean-Baptiste Donnet, 3 bis rue Alfred Werner, 68093 Mulhouse, France

${ }^{3}$ Université de Haute-Alsace (UHA), Laboratoire de Chimie Organique et Bioorganique (COB), EA 4566, Ecole Nationale Supérieure de Chimie de Mulhouse (ENSCMu), Institut Jean-Baptiste Donnet, 3 bis rue Alfred Werner, 68093 Mulhouse, France

\section{Supplementary information}

Table of Contents:

1. General methods and materials

2. Experimental procedures and characterizations

3. References

4. ${ }^{1} \mathrm{H}$ and ${ }^{13} \mathrm{C}$ NRM of compounds 5-7, 1 at $295 \mathrm{~K}$

\section{1- General methods and materials :}

Reagents were purchased from commercial suppliers and used without further purification. $\mathrm{NEt}_{3}$ was freshly distilled from $\mathrm{KOH}$, THF from sodium/benzophenone, and 
$\mathrm{CHCl}_{3}$ from $\mathrm{P}_{2} \mathrm{O}_{5} . \mathrm{CH}_{3} \mathrm{CN}$ and propionitrile were dried over $3 \AA$ molecular sieves. IR spectra $\left(\mathrm{cm}^{-1}\right)$ were recorded on either a Bruker Vertex 70 or an Equinox 55 spectrometer. ${ }^{1} \mathrm{H}$ NMR (400 MHz) and ${ }^{13} \mathrm{C}$ NMR (100.6 MHz) spectra were measured with a Bruker Avance (Serie 400). ${ }^{1} \mathrm{H}$ and ${ }^{13} \mathrm{C}$ NMR spectra of compounds 6-7 have been recorded at $295 \mathrm{~K}$ but also at 329 $\mathrm{K}$ so as to improve their resolutions. They were then compared among themselves to facilitate their interpretations. High resolution MS were measured with an Agilent Technologies 6510 (Q-TOF) Spectrometer using a dual ESI source.

\section{2- Experimental procedures and characterizations}

tert-Butyl-1,7-diaza-(15-crown-5)-1-carboxylate 2:

A solution of 1,7-diaza-15-crown-5 (1.0 g, $4.6 \mathrm{mmol}$.) and di-tert-butyl dicarbonate (1.09 g, 1 equiv.) in $\mathrm{CH}_{3} \mathrm{CN}$ (15 mL) was stirred at rt, for $16 \mathrm{~h} . \mathrm{CH}_{3} \mathrm{CN}$ was then evaporated under reduced pressure. Purification by column chromatography (acetone/ $\mathrm{NEt}_{3} \quad 95: 5$ ) afforded the pure monoprotected compound $2(0.61 \mathrm{~g}, 42 \%)$ as an orange oil. ${ }^{1} \mathrm{H},{ }^{13} \mathrm{C}$ NMR and HRMS were in agreement with literature. ${ }^{1}$

\section{4-[(1-Aza-(15-crown-5)-1-yl)-4 oxo]butyric acid 3:}

A solution of 1-aza-15-crown-5 (1 g, $4.56 \mathrm{mmol}$ ), succinic anhydride (0.91 g, 2 equiv.) and $\mathrm{NEt}_{3}\left(2.5 \mathrm{~mL}, 4\right.$ equiv.) in $\mathrm{CH}_{3} \mathrm{CN}(2 \mathrm{~mL})$ was stirred at $\mathrm{rt}$, for $17 \mathrm{~h}$, under $\mathrm{Ar}$. $\mathrm{CH}_{3} \mathrm{CN}$ was then evaporated under reduce pressure. The crude product was dissolved in $\mathrm{CH}_{2} \mathrm{Cl}_{2}(20 \mathrm{~mL})$. This organic layer was successively extracted three times with aqueous $1 \mathrm{M}$ $\mathrm{HCl}$ and with $20 \%$ brine. The combined aqueous layers were extracted twice with $\mathrm{CH}_{2} \mathrm{Cl}_{2}(10$ $\mathrm{mL}$ ). The combined organic layers were then dried over $\mathrm{MgSO}_{4}$ and evaporated under

reduced pressure to afforded pure 3 as a yellowish semi-solid $(1.3 \mathrm{~g}, 89 \%) .{ }^{1} \mathrm{H},{ }^{13} \mathrm{C}$ NMR and HRMS were in agreement with literature. ${ }^{2}$

tert-Butyl-7[(1-aza(15-crown-5)-1-(1,4-dioxo-but-4-yl)]-1,7-diaza-15-crown-5)-1carboxylate 5: 
A solution of $3(100 \mathrm{mg}, 0.31 \mathrm{mmol}$ ) and $N, N$-dicyclohexylcarbodiimide $(64 \mathrm{mg}, 1$ equiv. $)$ in $\mathrm{CHCl}_{3}(0.4 \mathrm{~mL})$ was stirred at $\mathrm{rt}$ for $1 \mathrm{~h}$. Carbamate $2(120 \mathrm{mg}, 1.5$ equiv. $)$ and $\mathrm{NEt}_{3}$ (128 $\mu \mathrm{L}, 3$ equiv.) were successively added. The reaction mixture was stirred, at $\mathrm{rt}$, for $17 \mathrm{~h}$. Distilled water $(50 \mu \mathrm{L})$ was added and the reaction mixture was thoroughly vigorously stirred for $4 \mathrm{~h}$. The reaction mixture was then co-evaporated with toluene under reduce pressure. The obtained residue was treated three times with anhydrous toluene and the precipitated urea were discarded. The combined organic layers were evaporated under reduce pressure. Purification by column chromatography (acetone/ $\mathrm{NEt}_{3}$ ) afforded pure 5 as on orange oil (75 mg, 39\%). ${ }^{1} \mathrm{H}$ NMR (400 MHz, $\left.\mathrm{CDCl}_{3}\right): 1.44$ (s, 9H, $\left.3 \mathrm{CH}_{3}\right), 2.70$ (m, 4H, NCOC$\left.\underline{H}_{2}\right), 3.60-$ $3.90\left(\mathrm{~m}, 40 \mathrm{H}, 6 \mathrm{NCH}_{2}, 14 \mathrm{OC}_{2}\right)$ ppm. ${ }^{13} \mathrm{C} \mathrm{NMR}\left(100.6 \mathrm{MHz}, \mathrm{CDCl}_{3}\right): \delta=28.3\left(3 \mathrm{C}, 3 \underline{\mathrm{CH}}_{3}\right)$, $[28.5,29.1]\left(2 \mathrm{C}, \mathrm{NCO}^{\mathrm{C}} \mathrm{H}_{2}\right),[47.7,48.0,48.2,48.7,49.1,49.3,49.5,50.2]\left(6 \mathrm{C}, \underline{\mathrm{CH}}_{2} \mathrm{~N}\right),[68.6$, $69.1,69.3,69.4,69.5,70.0,70.1,70.4,70.5,70.6,70.9,71.3,71.8]\left(14 \mathrm{C}, \mathrm{OC}_{\mathbf{C}}\right), 79.5(1 \mathrm{C}$,

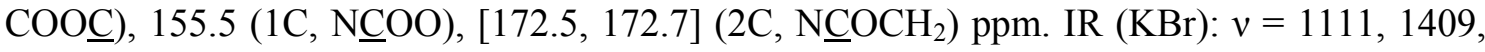
1459, 1637, 1687, 2867, 3400-3550 $\mathrm{cm}^{-1}$. HRMS (ESI-Q-Tof) : calcd for $\mathrm{C}_{29} \mathrm{H}_{53} \mathrm{~N}_{3} \mathrm{NaO}_{11}$ $[\mathrm{M}+\mathrm{Na}]^{+} 642.3572$; found 642.3574 .

\section{[(1-Aza-(15-crown-5)-1-(1,4-dioxa-but-4-but-4-yl)]-1,7-diaza-(15-crown-5)hydrochloride}

6:

Carboxylate 5 (290 mg, $0.47 \mathrm{mmol}$.) was dissolved in an anhydrous solution of $1.5 \mathrm{M}$ $\mathrm{HCl}$ in $\mathrm{Et}_{2} \mathrm{O}$ (1 mL, 5 equiv.). The reaction mixture was stirred, at $\mathrm{rt}$, for $18 \mathrm{~h}$. After evaporation of $\mathrm{Et}_{2} \mathrm{O}$, the pure hydrochloride 6 was recovered as yellow crystals $(260 \mathrm{mg}$, 100\%). ${ }^{1} \mathrm{H}$ NMR (400 MHz, $\left.\mathrm{CDCl}_{3}\right): 2.61$ (m, 4H, NCOC$\left.\underline{\mathrm{H}}_{2}\right), 3.0-3.33$ (m, 6H, $3 \mathrm{NC}_{2}$ ), 3.33-4.0 (m, 34H, $\left.3 \mathrm{NC}_{2}, 14 \mathrm{OC}_{2}\right), 9.08\left(\mathrm{~s}, 1 \mathrm{H}, \mathrm{N}_{2} \mathrm{Cl}\right), 9.25\left(\mathrm{~s}, 1 \mathrm{H}, \mathrm{N}_{2} \mathrm{Cl}\right) \mathrm{ppm} .{ }^{13} \mathrm{C}$ $\operatorname{NMR}\left(100.6 \mathrm{MHz}, \mathrm{CDCl}_{3}\right): \delta=[27.9,28.0]\left(2 \mathrm{C}, \underline{\mathrm{CH}_{2} \mathrm{CON}}\right),[47.2,47.5,47.8,48.4,49.0$, 49.2, 49.6, 50.0, 50.1] (4C, $\left.\underline{\mathrm{CH}}_{2} \mathrm{NCO}\right),[65.6,65.9]\left(2 \mathrm{C}, \underline{\mathrm{CH}}_{2} \mathrm{NH}_{2} \mathrm{Cl}\right),[67.9,68.6,69.1,69.3$, $69.7,69.8,69.9,70.0,70.5,71.0]\left(14 \mathrm{C}, \mathrm{C}_{2} \mathrm{O}\right),[172.2,172.7]\left(2 \mathrm{C}, \mathrm{NCOCH}_{2}\right)$ ppm. IR 
$(\mathrm{KBr}): v=748,1115,1355,1445,1518,1579,1636,3100-2500 \mathrm{~cm}^{-1}$. HRMS (ESI-Q-Tof) : calcd for $\mathrm{C}_{24} \mathrm{H}_{46} \mathrm{~N}_{3} \mathrm{O}_{9}[\mathrm{M}+\mathrm{H}]^{+} 520.3229$; found 520.3243 .

\section{\{7-[1-Aza-(15-crown-5)-1-(1,4-dioxo-but-4-yl)]-1-docosyl\}-1,7-diaza-(15-crown-5) 7 :}

A solution of 6 (245 mg, 0.44 mmol.), bromodocosane (172 mg, 1 equiv.) and $\mathrm{Na}_{2} \mathrm{CO}_{3}$ (94 mg, 2 equiv.) in propionitrile $(25 \mathrm{~mL})$ was stirred, at $100^{\circ} \mathrm{C}$, for $22 \mathrm{~h}$. Propionitrile was evaporated under reduced pressure. Purification by column chromatography (acetone/ $\mathrm{NEt}_{3}$ $95: 5)$ afforded the pure 7 compound (120 mg, 33\%) as a yellow oil. ${ }^{1} \mathrm{H}$ NMR (400 MHz, $\left.\mathrm{CDCl}_{3}\right): 0.88\left(\mathrm{t},{ }^{3} J=6.8 \mathrm{~Hz}, 3 \mathrm{H}, \mathrm{C}_{3}\right), 1.25\left(\mathrm{bs}, 38 \mathrm{H}, \underline{\mathrm{CH}}_{2}(3-21)\right), 1.35-1.50\left(\mathrm{~m}, 2 \mathrm{H}, \underline{\mathrm{C}}_{2}(2)\right)$, 2.45 (bt, $\left.2 \mathrm{H},{ }^{3} J=6.8 \mathrm{~Hz}, \mathrm{NC}_{2}(1)\right), 2.65-2.83\left(\mathrm{~m}, 4 \mathrm{H}, \mathrm{NCOC}_{2}\right), 2.65-2.83\left(\mathrm{~m}, 4 \mathrm{H}, \mathrm{NC}_{2}\right)$, 3.50-3.85 (m, 36H, $\left.28 \mathrm{OCH}_{2}, 8 \mathrm{NCH}_{2}\right)$ ppm. ${ }^{13} \mathrm{C}$ NMR $\left(100.6 \mathrm{MHz}, \mathrm{CDCl}_{3}\right): \delta=14.1$ $(C(22)), 22.7(C(21)), 27.4(C(3)), 28.4(C(2))$, [29.3, 29.6, 29.7, 30.9, 31.9] (16C, C(4-19)), $[48.8,49.4,49.7,50.3]$ ( 4C, $\left.\underline{\mathrm{CH}}_{2} \mathrm{NCO}\right),[53.9,54.4]\left(2 \mathrm{C}, \mathrm{NC}_{2}\right), 56.8(\mathrm{~N} \underline{\mathrm{C}}(1)),[69.0,69.4$,

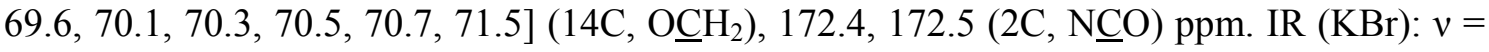
720, 1119, 1293, 1355, 1467, $1642 \mathrm{~cm}^{-1}$. HRMS (ESI-Q-Tof) : calcd for $\mathrm{C}_{46} \mathrm{H}_{89} \mathrm{~N}_{3} \mathrm{NaO}_{9}$ $[\mathrm{M}+\mathrm{Na}]^{+} 850.6491$; found 850.6494 .

\section{\{7-[1-Aza-(15-crown-5)-1-(butyl-4-yl)]-1-docosyl\}-1,7-diaza-(15-crown-5) 1:}

A suspension of 7 (563 mg, $0.68 \mathrm{mmol}$.) and $\mathrm{LiAlH}_{4}$ (100 mg, 4 equiv.) in THF (18 $\mathrm{mL}$ ) was stirred for $18 \mathrm{~h}$. To the reaction mixture, $\mathrm{Na}_{2} \mathrm{SO}_{4}, 10 \mathrm{H}_{2} \mathrm{O}(1.5 \mathrm{~g})$ was added portionwise and then an aqueous $1 \mathrm{M} \mathrm{NaOH}(0.5 \mathrm{~mL})$ solution. AcOEt $(10 \mathrm{~mL})$ was added and the mixture was vigorously stirred for $20 \mathrm{~h}$ before extraction. The recovered organic layer was centrifugated. This procedure was repeated three times. The organic layers were combined and the solvents were evaporated under reduced pressure. The crude product was then dissolved in an anhydrous solution of $\mathrm{Et}_{2} \mathrm{O} .5 \mathrm{M} \mathrm{HCl}$ in $\mathrm{Et}_{2} \mathrm{O}$ was thoroughly added. The pure trihydrochloride 1 crystallized and was recovered by filtration $(431 \mathrm{mg}, 70 \%) .{ }^{1} \mathrm{H}$ NMR (400 MHz, $\left.\mathrm{CDCl}_{3}\right): 0.84\left(\mathrm{t},{ }^{3} \mathrm{~J}=6.8 \mathrm{~Hz}, 3 \mathrm{H}, \mathrm{C}_{3}\right), 1.26\left(\mathrm{bs}, 42 \mathrm{H}, \underline{\mathrm{C}}_{2}(3-21), \mathrm{NCH}_{2} \mathrm{C}_{2}\right)$, 


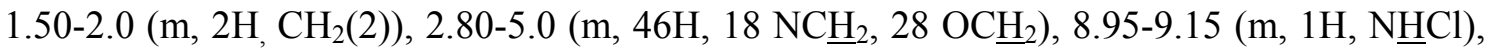
$9.82(\mathrm{~s}, 1 \mathrm{H}, \mathrm{NHCl}), 10.37(\mathrm{~s}, 1 \mathrm{H}, \mathrm{NHCl}) \mathrm{ppm} .{ }^{13} \mathrm{C} \mathrm{NMR}\left(100.6 \mathrm{MHz}, \mathrm{CDCl}_{3}\right): \delta=14.02$ (C(22)), $21.7(\mathrm{C}(21)), 25.8(\mathrm{C}(3)), 26.7(\mathrm{C}(2)),[28.9,29.2,29.3,29.4,29.5,29.5,29.6](18 \mathrm{C}$, $\left.\mathrm{C}(4-19), \underline{\mathrm{CCH}}_{2} \mathrm{~N}\right), 30.9(\mathrm{C}(20)),[47.4,47.5,47.7,52.2,53.0,53.4,54.1,54.5]\left(9 \mathrm{C}, \mathrm{NCH}_{2}\right)$, $[63.9,64.1,65.0,65.6,66.0,69.0,69.1,69.5,69.7,69.9,70.1,70.3,70.5]\left(14 \mathrm{C}, \mathrm{OC}_{2}\right) \mathrm{ppm}$. IR (KBr): $v=721,1100,1125,1467,1640,3390 \mathrm{~cm}^{-1}$. HRMS (ESI-Q-Tof) : calcd for $\mathrm{C}_{46} \mathrm{H}_{93} \mathrm{~N}_{3} \mathrm{NaO}_{2}[\mathrm{M}+\mathrm{Na}]^{+} 822.6906$; found 822.6916 .

\section{3- References}

1. a) Kumar, A.; Mageswaran, S.; Sutherland, I. O. Tetrahedron 1986, 12, 3291. b) Jones, N. F. ; Kumar, A.; Sutherland, I.O. J. Chem. Soc. Chem. Commun. 1981, 19, 990.

2. Jiang, L.; Liu, Z.; Liang, Z.; Gao, Y. Bioorg. Med. Chem. 2005, 11, 3673.

\section{4- ${ }^{\mathbf{1}} \mathbf{H}$ and ${ }^{13} \mathbf{C}$ NMR at $295 \mathrm{~K}$}


i̊

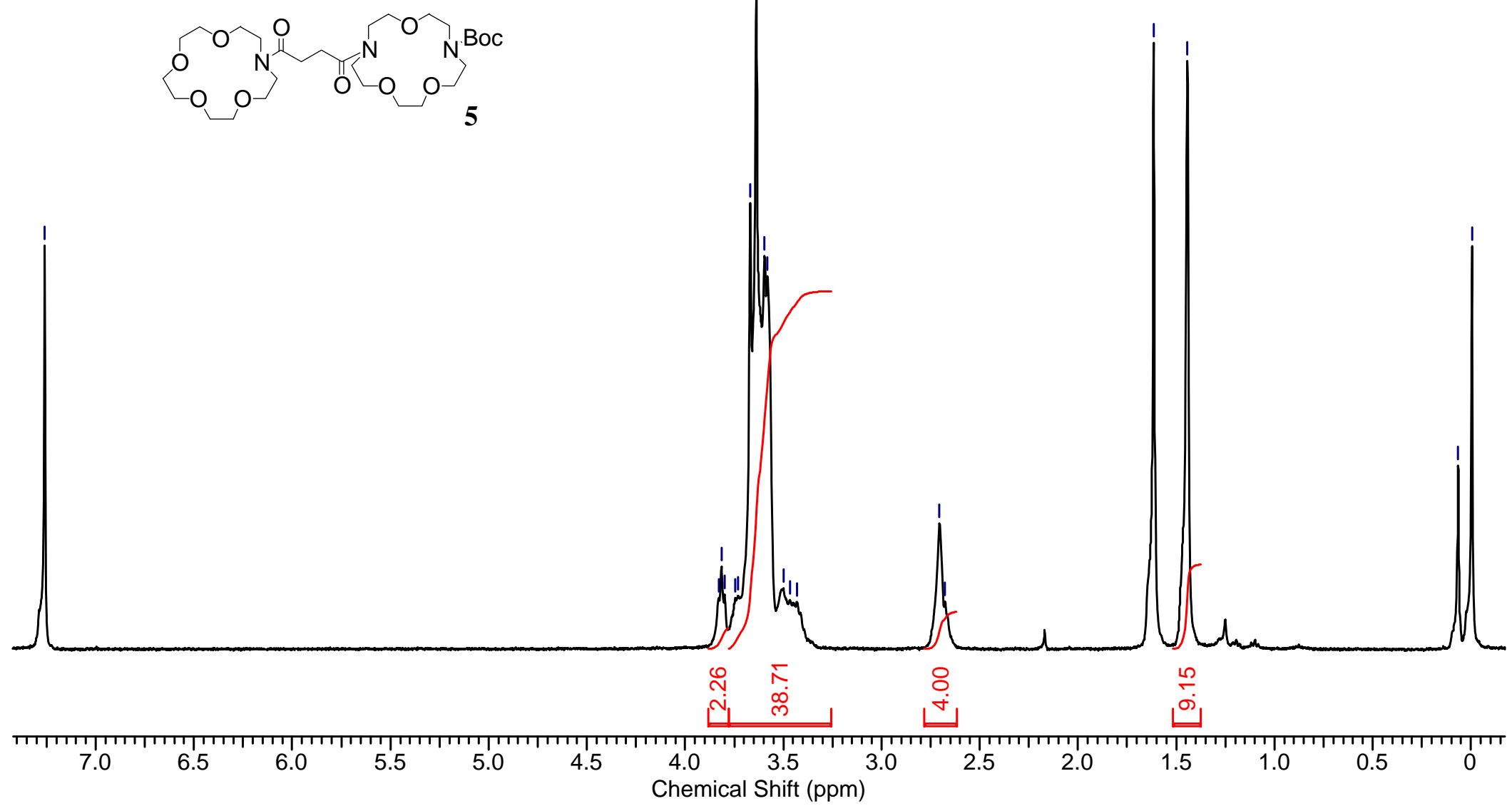

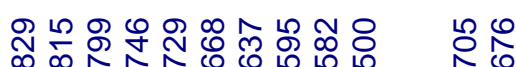
लं लं लं

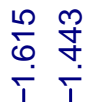

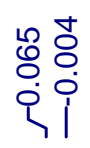




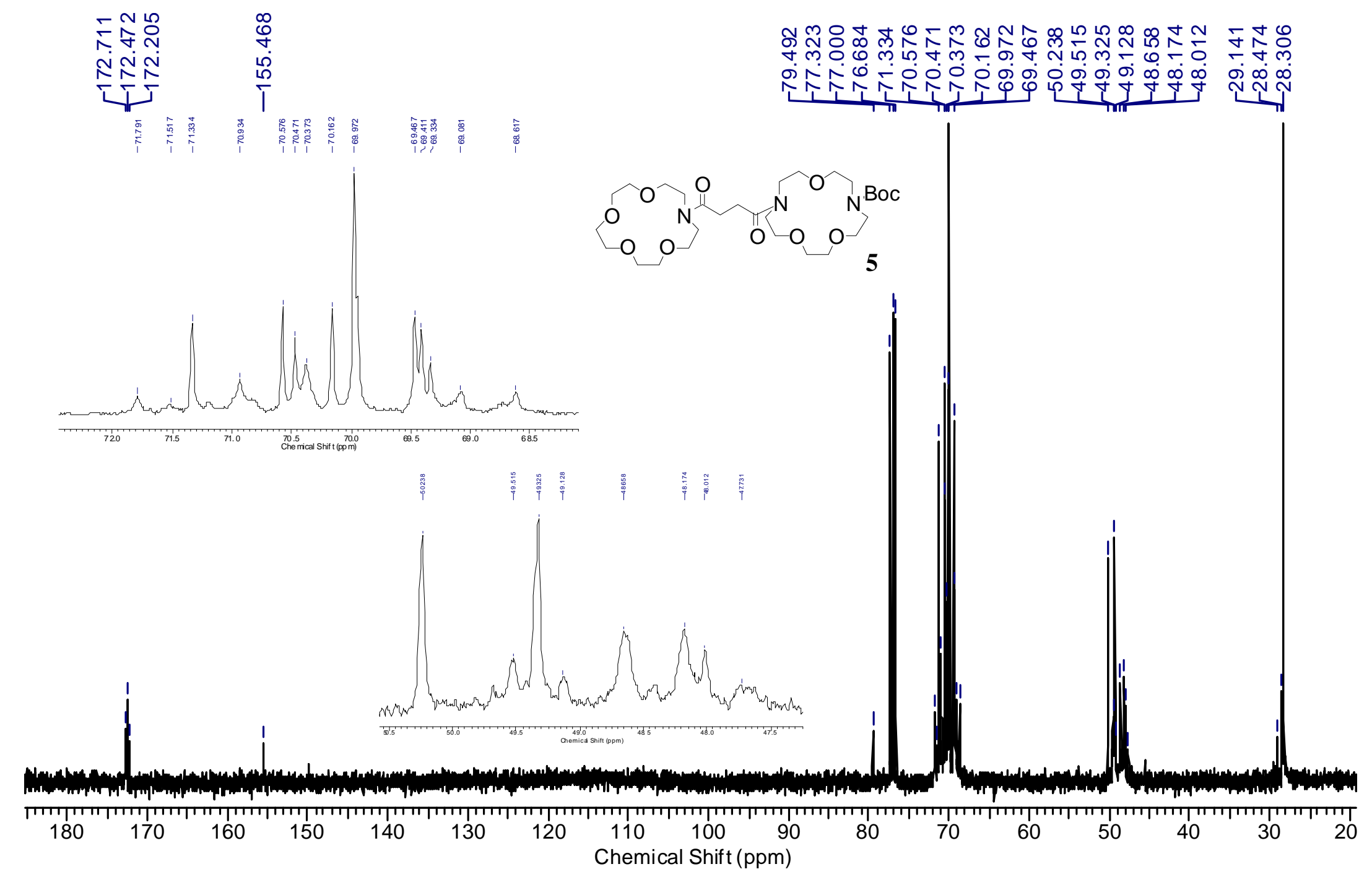




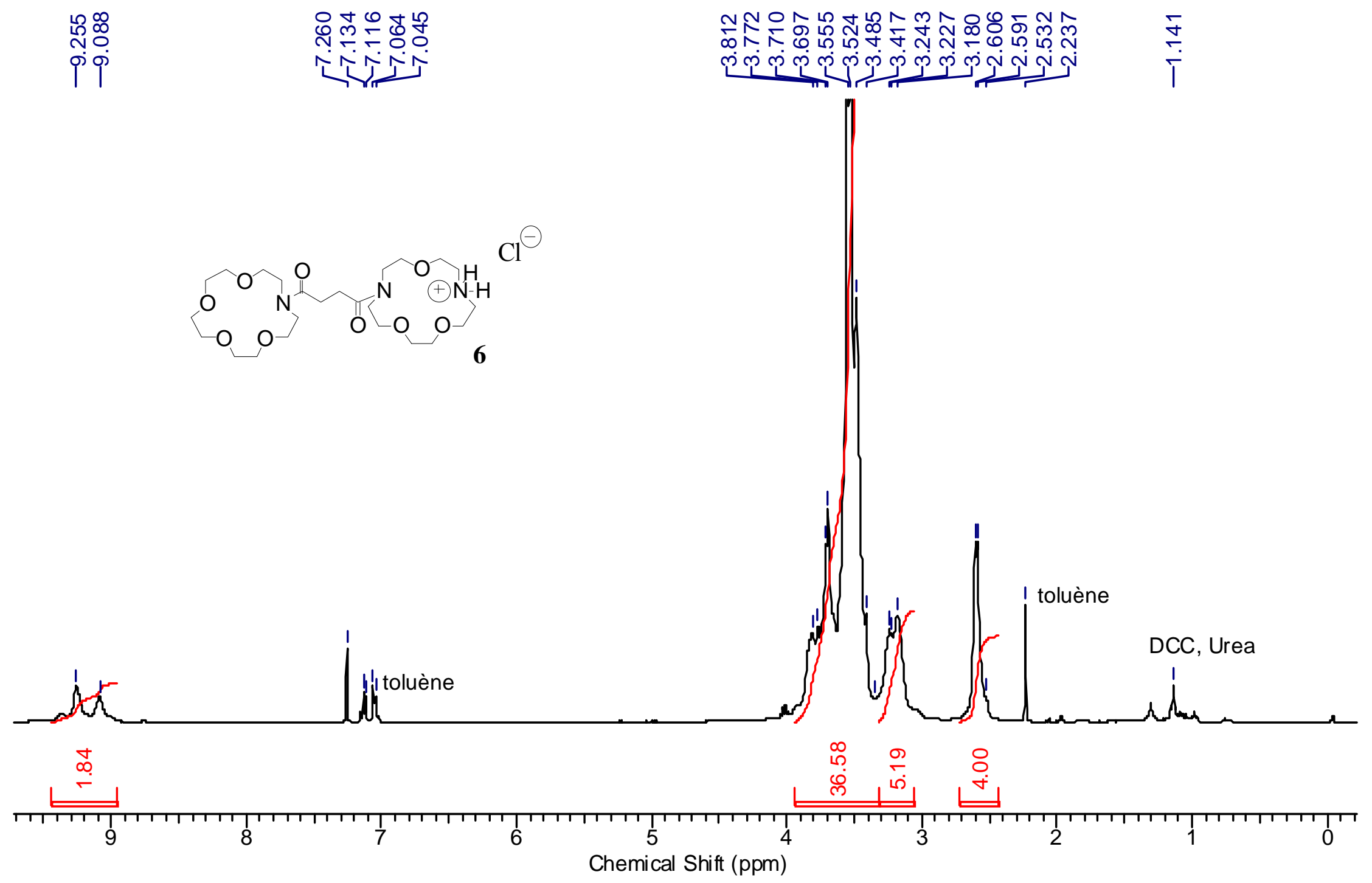




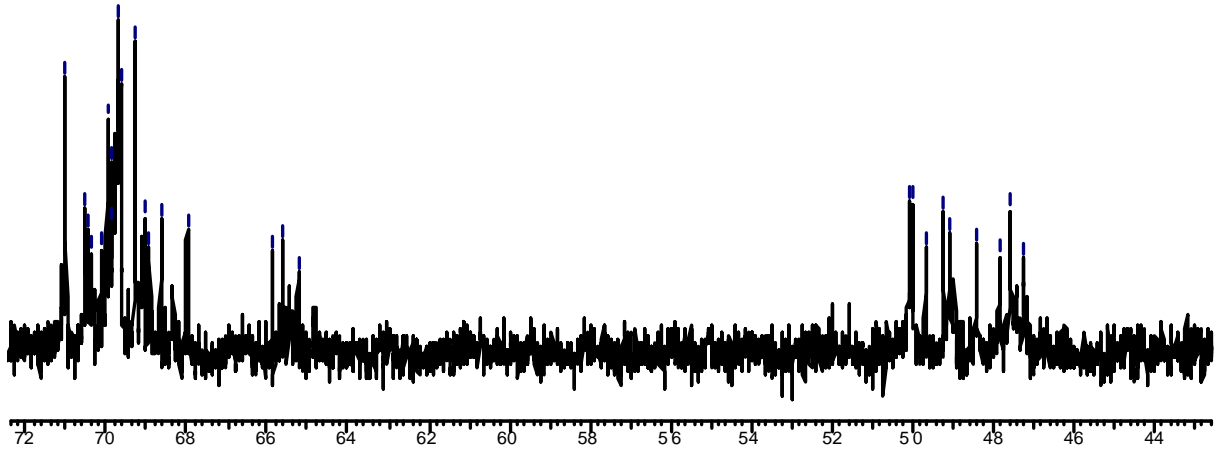

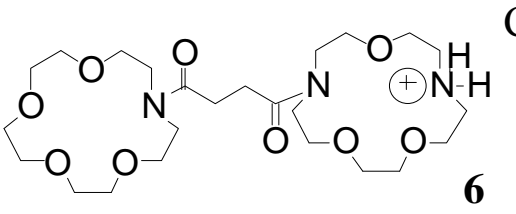

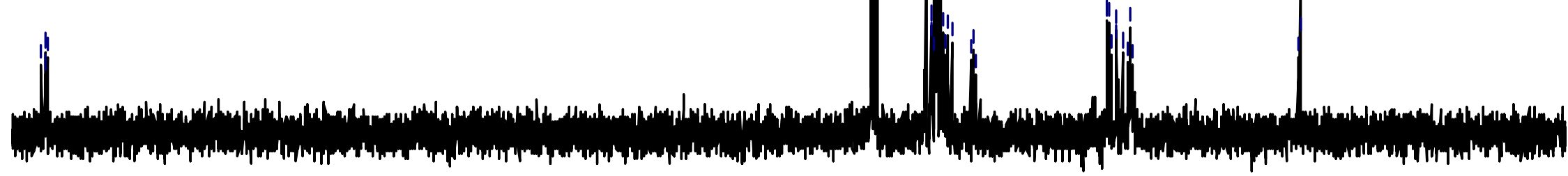

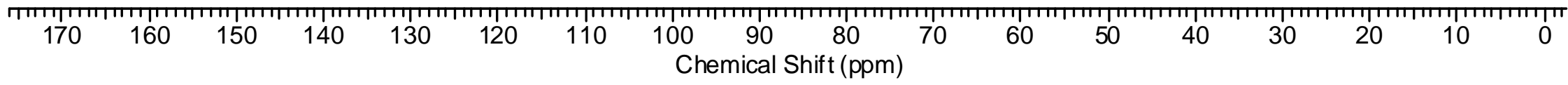




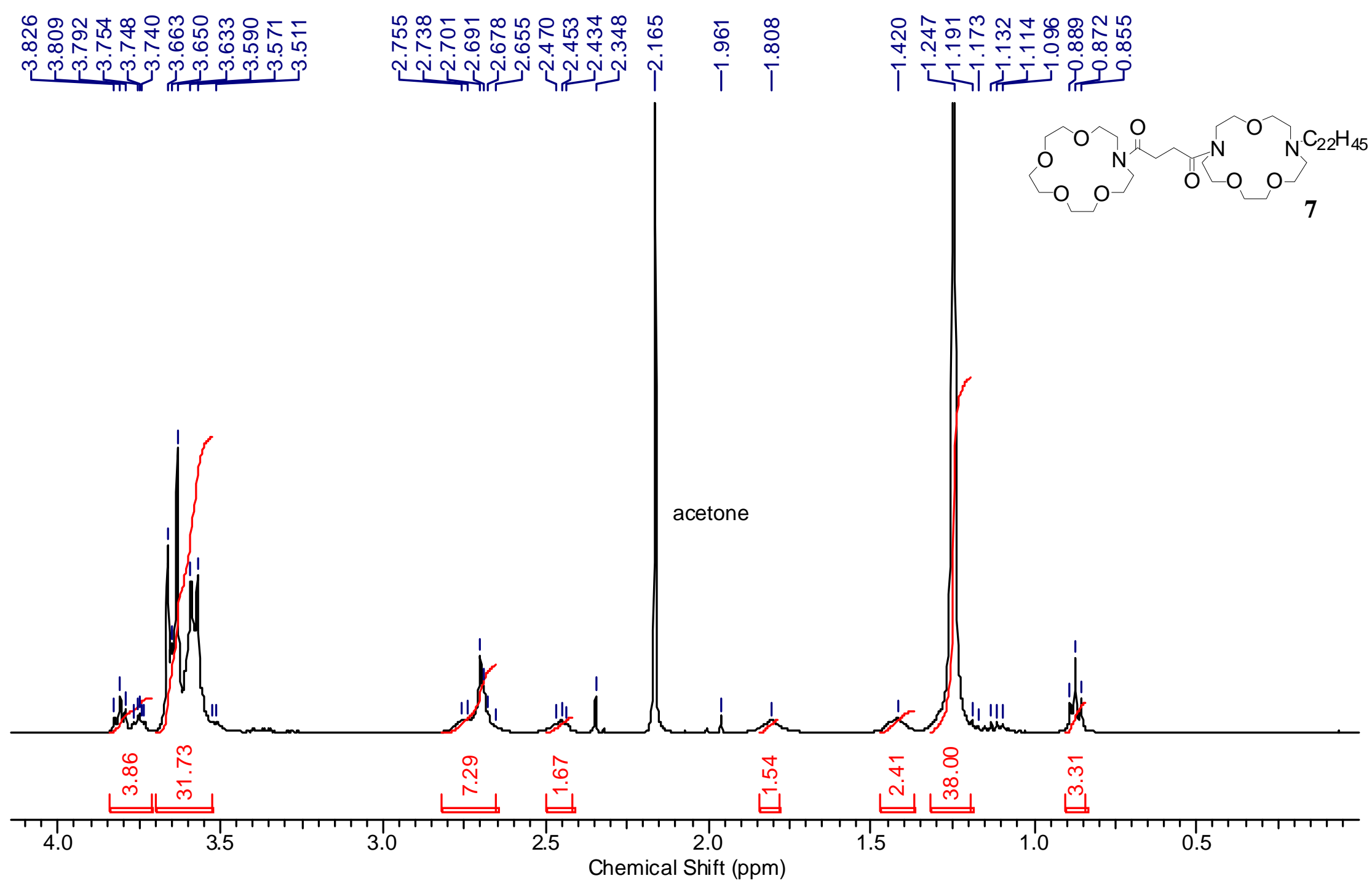




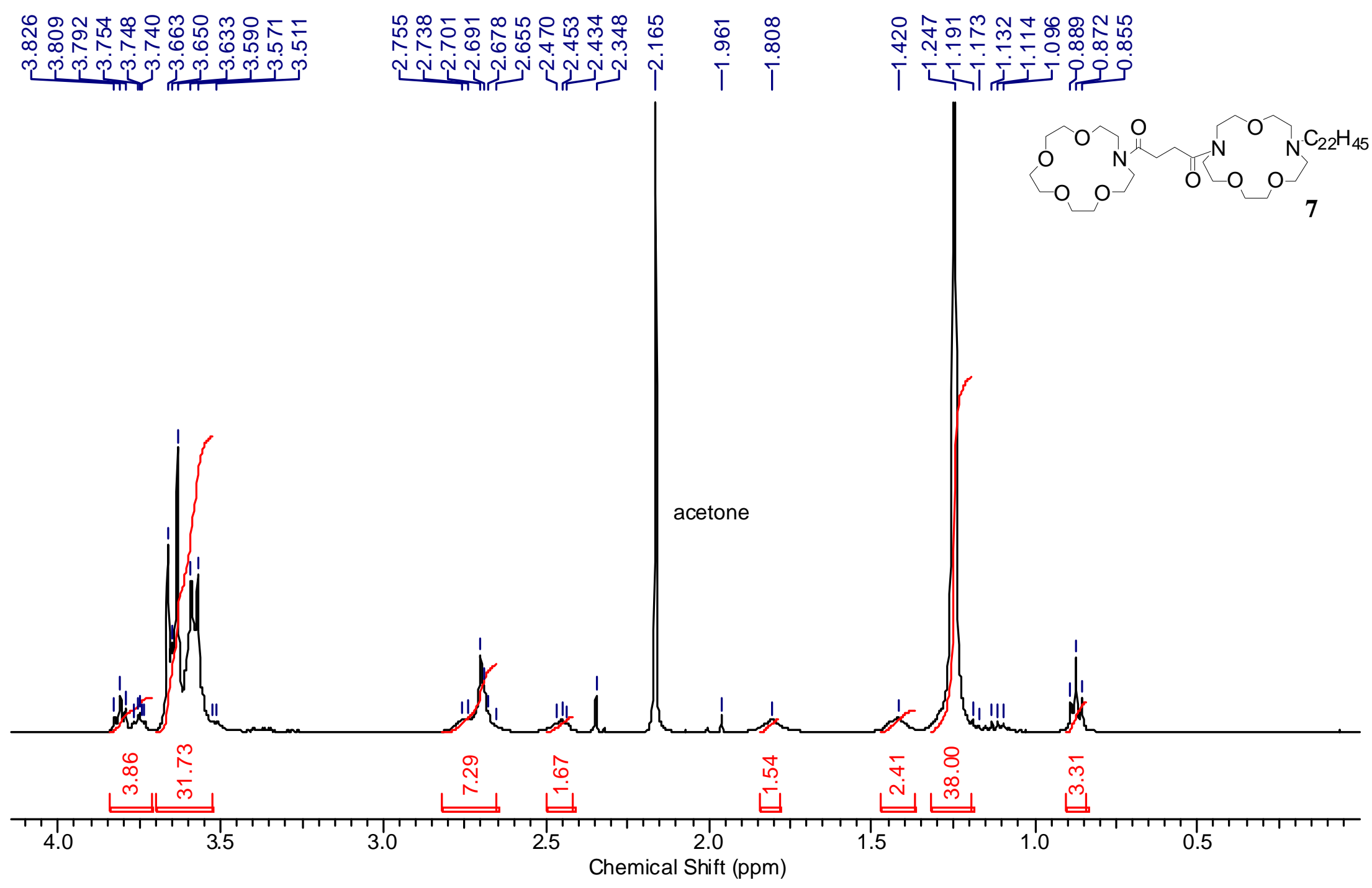




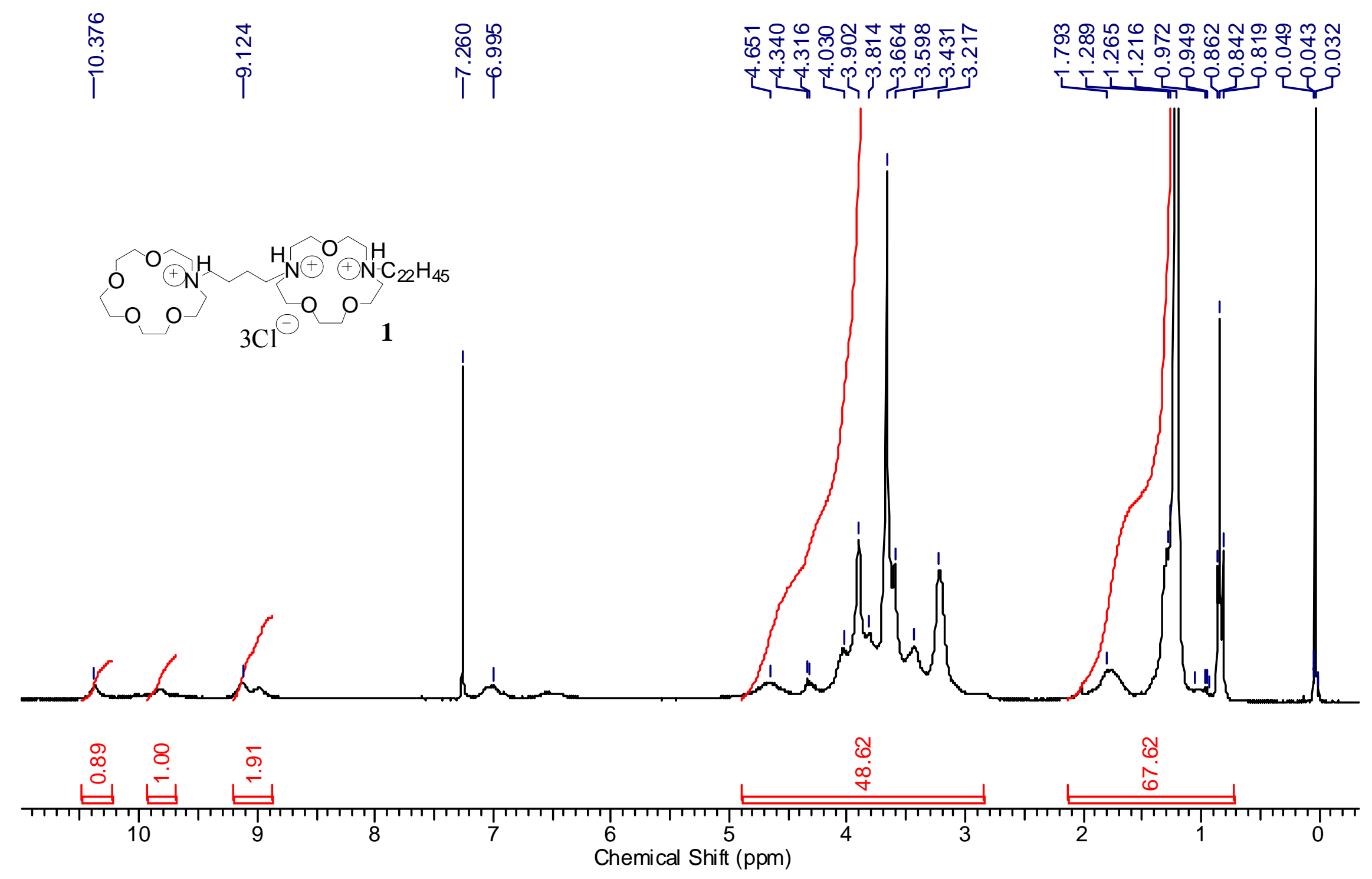




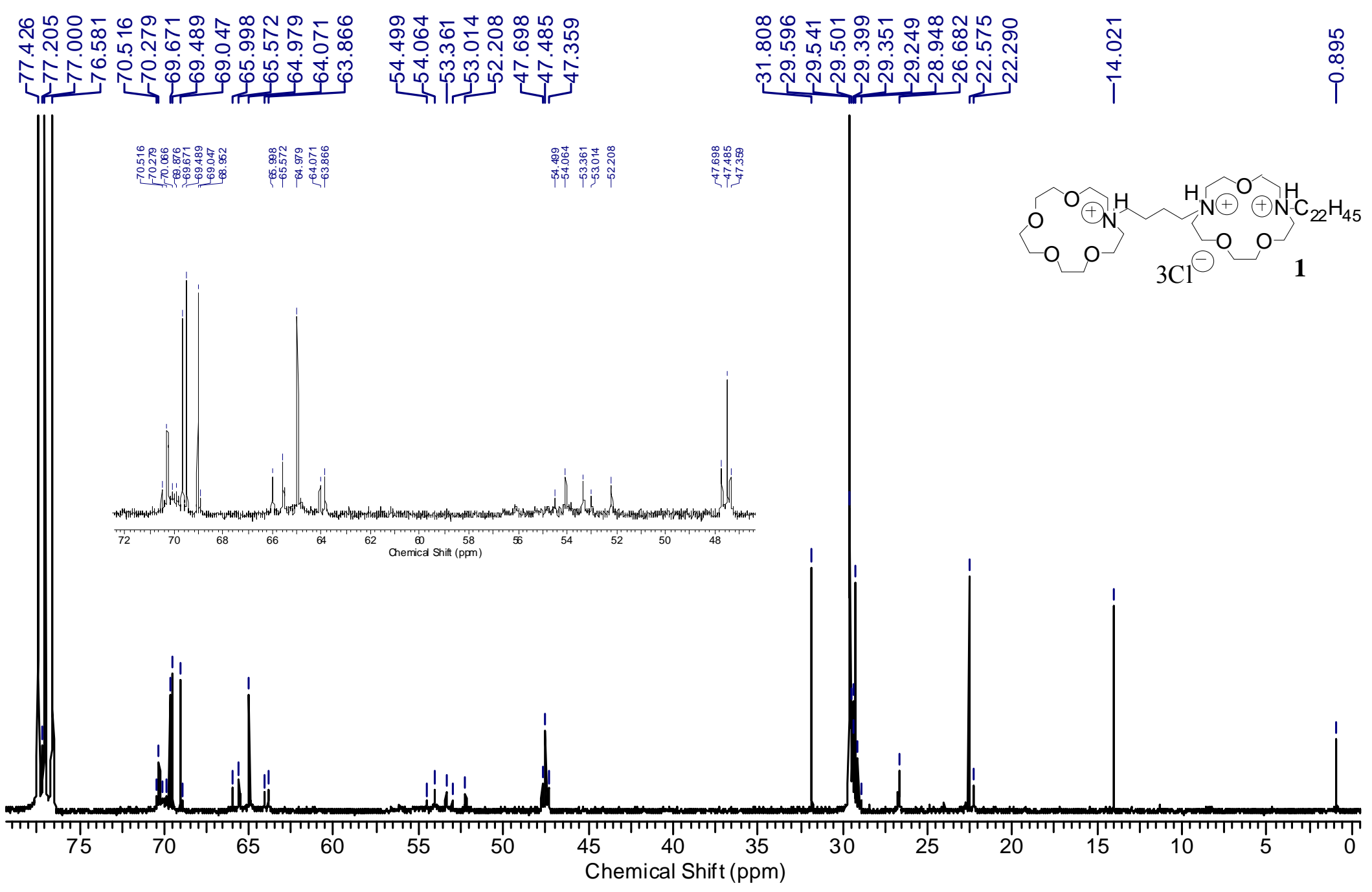


